\title{
Elimination of PCR duplicates in RNA-seq and small RNA-seq using unique molecular identifiers
}

\author{
Yu Fu ${ }^{1,2+}$, Pei-Hsuan $\mathrm{Wu}^{3 \dagger}$, Timothy Beane ${ }^{3}$, Phillip D. Zamore ${ }^{3^{*}}$ (10) and Zhiping Weng ${ }^{2,4^{*}}$
}

\begin{abstract}
Background: RNA-seq and small RNA-seq are powerful, quantitative tools to study gene regulation and function. Common high-throughput sequencing methods rely on polymerase chain reaction (PCR) to expand the starting material, but not every molecule amplifies equally, causing some to be overrepresented. Unique molecular identifiers (UMIs) can be used to distinguish undesirable PCR duplicates derived from a single molecule and identical but biologically meaningful reads from different molecules.

Results: We have incorporated UMIs into RNA-seq and small RNA-seq protocols and developed tools to analyze the resulting data. Our UMIs contain stretches of random nucleotides whose lengths sufficiently capture diverse molecule species in both RNA-seq and small RNA-seq libraries generated from mouse testis. Our approach yields high-quality data while allowing unique tagging of all molecules in high-depth libraries.

Conclusions: Using simulated and real datasets, we demonstrate that our methods increase the reproducibility of RNA-seq and small RNA-seq data. Notably, we find that the amount of starting material and sequencing depth, but not the number of PCR cycles, determine PCR duplicate frequency. Finally, we show that computational removal of PCR duplicates based only on their mapping coordinates introduces substantial bias into data analysis.
\end{abstract}

Keywords: RNA-seq, Small RNA-seq, Unique molecular identifier, UMI, PCR duplicates, PCR cycle, Starting material, Sequencing depth, Transcriptome, Ribognome

\section{Background}

High-throughput sequencing of long (>100 nt) or small (18-50 nt) RNA provides a quantitative measure of RNA abundance. However, RNA-seq and small RNA-seq library construction can introduce bias at multiple steps, such as fragmentation of long RNAs, reverse transcription, adapter ligation, library amplification by PCR, and sequencing. Commonly used high-throughput sequencing platforms, including those made by Illumina and Pacific Biosciences, require PCR amplification during

\footnotetext{
* Correspondence: phillip.zamore@umassmed.edu;

zhiping.weng@umassmed.edu

${ }^{\dagger}$ Yu Fu and Pei-Hsuan Wu contributed equally to this work.

${ }^{3}$ RNA Therapeutics Institute and Howard Hughes Medical Institute, University of Massachusetts Medical School, 368 Plantation Street, Worcester, MA 01605, USA

${ }^{2}$ Program in Bioinformatics and Integrative Biology, University of

Massachusetts Medical School, 368 Plantation Street, Worcester, MA 01605, USA

Full list of author information is available at the end of the article
}

library construction to increase the number of cDNA molecules to an amount sufficient for sequencing. However, PCR stochastically introduces errors that can propagate to later cycles $[1,2]$. PCR also amplifies different molecules with unequal probabilities [1]. PCR duplicates are reads that are made from the same original cDNA molecule via PCR.

A common practice to eliminate PCR duplicates is to remove all but one read of identical sequences, assuming that such reads have been created from the same cDNA molecule by PCR [3]. This assumption may be flawed, especially with ever higher sequencing throughput, which increases the chance of observing reads with identical sequences but from different cDNA molecules. The situation is further exacerbated for small genomes and for techniques that interrogate a subspace of the genome. For example, the majority of small RNA-seq reads are microRNAs (miRNAs) or PIWI-interacting RNAs (piRNAs), which derive from loci that amount to just a 
few percent of the genome [4-7]. The assumption also has systematic biases. For example, a shorter gene is more likely to give rise to identical RNA-seq reads than a longer gene with the same transcript level, simply because the "genomic space" for the random process of RNA fragmentation is smaller for the shorter gene. Finally, the conventional definition of PCR duplicates is based on mapping coordinates-reads mapping to the exact same genomic location are considered to have identical sequences. However, many small RNAs with the same sequence can be produced from multiple genomic loci; thus, strategies using genome mapping to identify PCR duplicates ignore the situation that identical reads arise from distinct sites in the genome.

Standard library preparation and sequencing procedures typically have pre-specified PCR and sequencing error rates, but parameters such as the amount of starting RNA used to generate a library, the number of reads sequenced (i.e., sequencing depth), and the number of PCR cycles used are often adjusted to accommodate sample source, abundance, and quality. While the notion that more PCR amplification increases artefactual duplicate reads in high-throughput sequencing makes intuitive sense and is widely accepted, high PCR cycle numbers are often necessitated by scarce starting materials, another likely cause for duplicate reads. Thus, the contribution of PCR cycle number to PCR duplicates is often confounded with the contributions of starting materials and sequence depth.

Unique molecular identifiers (UMIs) are often used to accurately detect PCR duplicates and quantify transcript abundance [8-16]. If each molecule in the starting pool is barcoded with a UMI, i.e., all molecules are unique, then reads with the same UMI must be PCR duplicates. In practice, only the molecules in the starting pool that have identical sequences need to have different UMIs.

One strategy to incorporate UMIs introduces pre-defined, manually-selected sequences into the adapters. This strategy can avoid UMIs with suboptimal GC content and minimize complementarity between or within UMI sequences [13]. Because UMI identities are unambiguously defined, sequencing and PCR errors can be easily corrected. However, implementing pre-defined UMIs requires a large number of costly, custom-synthesized oligonucleotides.

An alternative strategy employs adapters that contain random nucleotides at certain positions in the adapters. The combinations of the random-nucleotide positions lead to an exponential number of different UMIs at almost no extra cost, because incorporating a random nucleotide costs the same as incorporating a specific nucleotide during DNA synthesis. UMIs bearing either five $\left(4^{5}=1,024\right.$ unique barcodes) or ten random nucleotides $\left(4^{10}=1,048,576\right.$ unique barcodes) were implemented cost-effectively and shown to improve PCR duplicate removal $[8,14]$. A higher number of unique combinations can be achieved simply by increasing the number of random-nucleotide positions. The number of UMI combinations must be sufficiently large because as mentioned above, the chance that two cDNA molecules with identical sequences in the starting pool are tagged with the same UMI combination needs to be infinitesimally small.

Here, we describe novel experimental protocols and computational methods to unambiguously identify PCR duplicates in RNA-seq and small RNA-seq data. We show that removing PCR duplicates using UMI information is accurate, whereas removing PCR duplicates without UMIs is overly aggressive, eliminating many biologically meaningful reads. Finally, we show that the amount of starting materials and sequencing depth determine the level of PCR duplicates, without additional contribution from the extent of PCR amplification.

\section{Results \\ Adapting standard RNA-seq procedures to incorporate UMIs}

To incorporate UMIs into RNA-seq, we modified a published, strand-specific, library construction protocol [17]. The original method has proved to be robust and time-efficient, and the adapter ligation step uses DNA adapter oligonucleotides that can be readily synthesized at a low cost $[7,18-20]$. The standard protocol uses a single Y-shaped DNA adapter comprising two partially complementary oligonucleotides and an unpaired 3 ' thymidine that pairs with the single adenine tail added to both ends of the double-stranded cDNA fragments. We modified the adapters by inserting a five-nucleotide random UMI (Fig. 1a, b). Consequently, each cDNA fragment is ligated to an adapter with a UMI at each end, randomly choosing one out of 1,048,576 $\left(4^{5} \times 4^{5}\right)$ possible combinations provided by two UMIs.

Our UMI RNA-seq adapters were designed so that the sequencing reaction begins at the very first nucleotide of the 5' UMI (Fig. 1b). The random nucleotides of UMIs offer the sequence diversity in the initial five sequencing cycles. This sequence diversity is critical for commonly used Illumina sequencing platforms, such as HiSeq, MiSeq, and NextSeq, to generate base-calling templates and make accurate models for discriminating read clusters [21, 22]. To avoid insertions or deletions within or flanking a UMI, albeit rare, from altering the UMI identity, we further designed a "UMI locator", a pre-defined trinucleotide $3^{\prime}$ to the UMI (e.g. 5'-NNNNNATC-3'). The trinucleotide serves as an anchor allowing unambiguous identification of each UMI (Fig. 1b). Taking the properties of our sequencing instrument of choiceNextSeq 500-into consideration, the 3 nt UMI locator sequence and the mandatory thymidine required for ligation that immediately follows (Fig. 1b) corresponded 


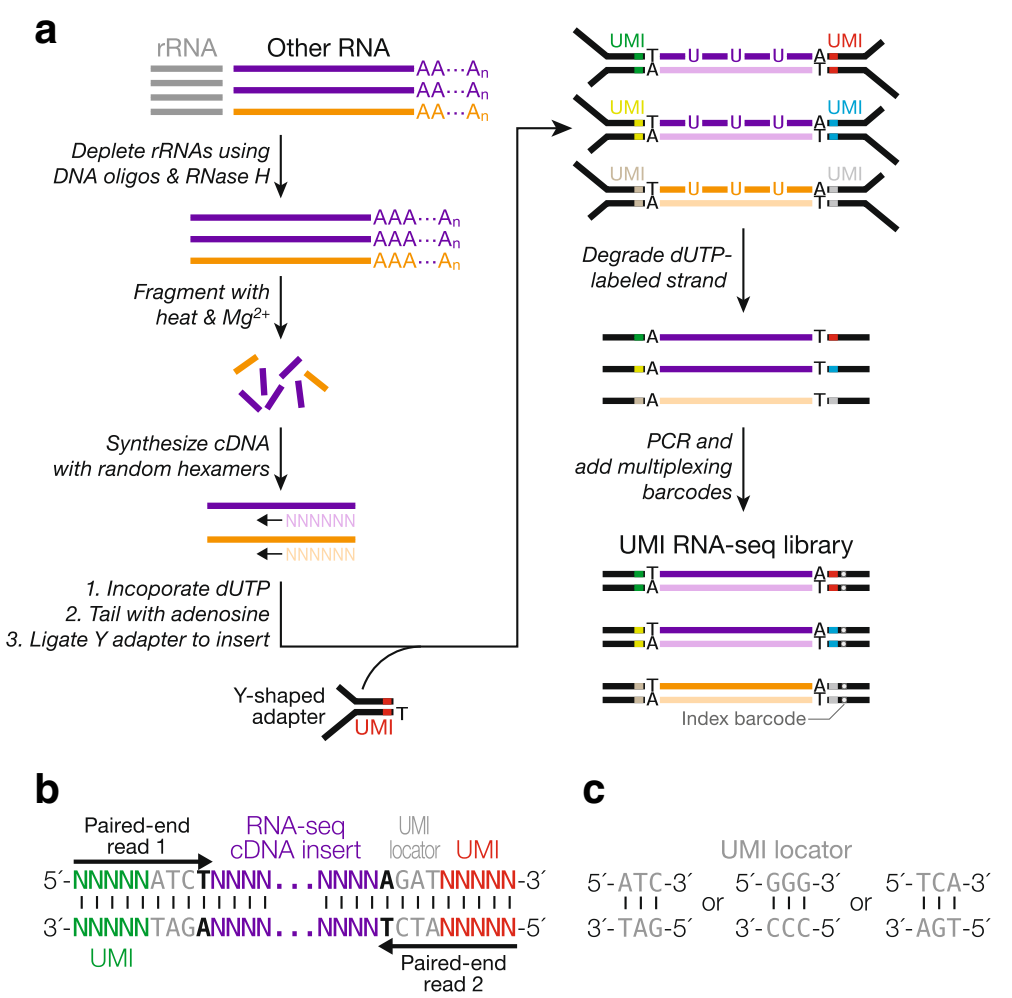

Fig. 1 UMI incorporation into RNA-seq. a Overall workflow. Schematic of a read produced from RNA-seq with UMIs (b) and of UMI locators (c)

to the sequencing cycles 6-9, after the first five critical cycles required by the instrument for template generation [23]. However, NextSeq still deemed these four invariant positions of low complexity and reported low-quality sequencing data. Previous approaches to tackle this problem include increasing the diversity of the initial sequences in the library, mixing the library with a high diversity sample (spike-in), lowering sequencing cluster density, or any combination of the above [21]. We designed three UMI locator sequences (Fig. 1c), and, by pooling adapters with one of these sequences at equimolar amounts, we were able to resolve the low complexity problem. Using this approach, we generated RNA-seq libraries from mouse brain, heart, kidney, liver, lung, muscle, spleen, and testis total RNAs. The libraries were sequenced at a read depth, coverage, and quality comparable to libraries generated using the original protocol without UMIs (Additional file 1). Thus, our method of incorporating UMIs, as well as UMI locator, does not interfere with library preparation and sequencing. We subsequently observed that even two different UMI locator sequences sufficed to overcome the erroneous low-quality calling by NextSeq (small RNA-seq, Fig. 2).

\section{Adapting standard small RNA-seq protocol to incorporate UMIs}

Previously, we established a reliable and robust small RNA-seq protocol by modifying a published method which utilizes oligonucleotides compatible with Illumina sequencing platforms [24, 25]. Compared to UMI RNA-seq, incorporation of UMIs into this small RNA-seq protocol requires additional considerations. First, the number of distinct UMI combinations needs to be significantly greater than what is required for RNA-seq. For example, millions of piRNA species-an abundant class of small RNAs in the animal germ line-can be routinely detected in a single individual, and it is estimated that there can be as many as 1 million distinct piRNA molecules in a single spermatocyte or round spermatid [4, 6, 26, 27]. The most abundant piRNA species in this study has 42,281 reads. In the soma, the most abundant miRNA can take up $>40 \%$ of the total sequencing depth [28] -tens of millions of reads. Such enormous abundance requires a sufficiently high number of UMI combinations to capture all distinct sequences. Second, the length range of small RNAs $(<50 \mathrm{nt})$ plus a longer UMI is still well within the read length achievable by common sequencing instruments. Third, the length of a small RNA is a defining feature of its identity and thus, insertions or deletions could lead to misclassification of small RNAs. The second and third considerations also indicate that small RNA-seq is ideally suited for the testing of a large combination of UMIs.

We tested UMIs containing 10 consecutive random nucleotides. Although both the 3' and 5' adapters containing 10-nt UMIs ligated to small RNAs with nearly 


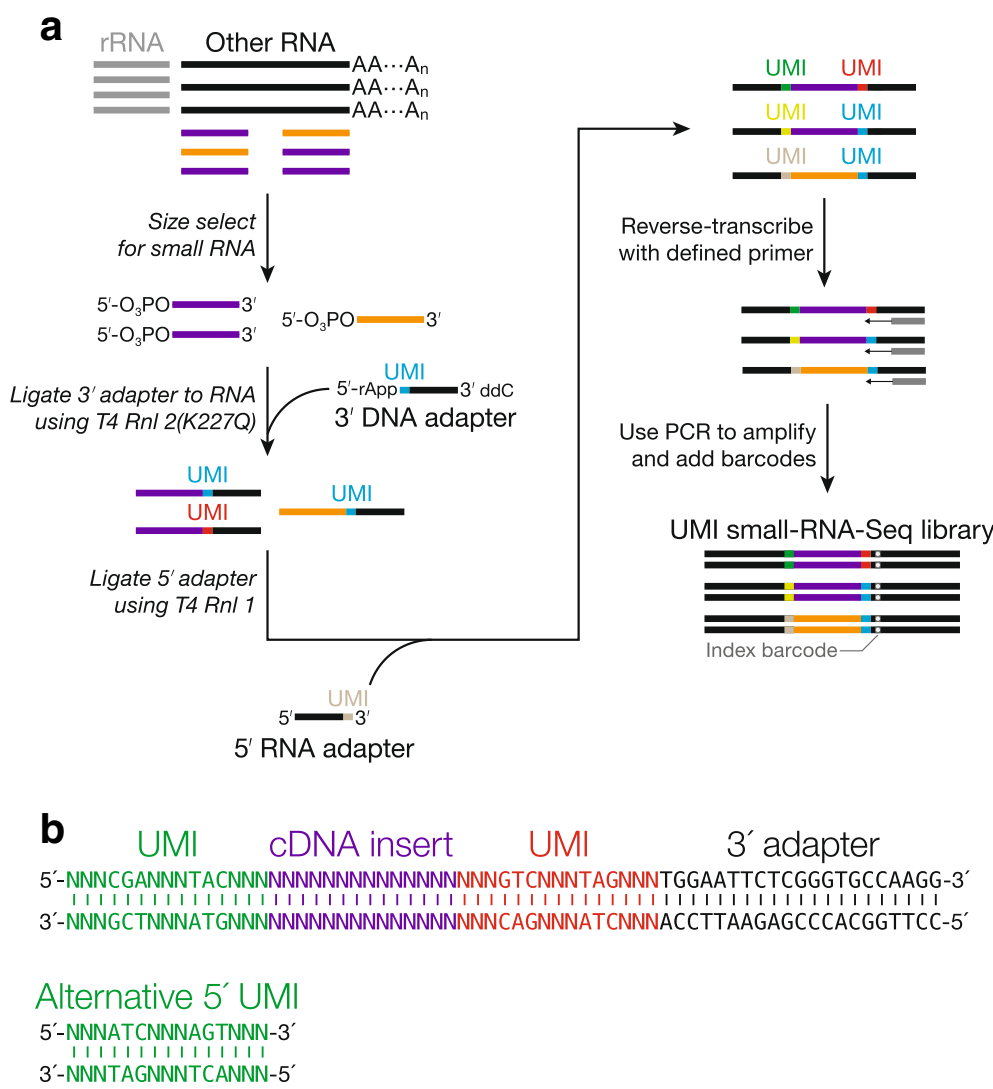

Fig. 2 UMI incorporation into small RNA-seq. a Overall workflow. The method uses a 3' adapter composed of DNA, except for a single, 5' ribonucleotide $(\mathrm{rA})$; the $5^{\prime}$ adapter is entirely RNA. A standard index barcode allows multiplexing. b Schematic of a read produced from small RNA-seq with UMIs

the same efficiency as the original adapters without UMIs, the resulting small RNA-seq libraries yielded unexpectedly short, variable-length reads that contained truncated insert and adapter sequences (data not shown). We speculate that long stretches of random nucleotides interfere with oligonucleotide annealing, a critical step in cDNA synthesis, PCR, and sequencing, by increasing the chance that a primer anneals to a UMI instead of its target sequences. Inter- and intramolecular annealing of $10 \mathrm{nt}$ UMIs may also contribute to truncated reads.

To avoid a long stretch of random nucleotides, we used the UMI locator strategy described above to space out several short stretches of random nucleotides. For each adapter, we designed three trinucleotide UMI sequences, each separated from another by a trinucleotide UMI locator (e.g., 5'-NNN-CGA-NNN-TAC-NNN-3'; Fig. 2a, b). Two adapters with such UMIs can produce a trillion combinations, which should suffice all deep-sequencing applications. Similar to our RNA-seq strategy, we designed adapters with two different sets of UMI locator sequences-mixed at equimolar-to increase the sequence complexity in the early sequencing cycles. This strategy allowed us to successfully generate and sequence the UMI small RNA-seq libraries, unambiguously locate UMIs, and computationally remove reads containing insertions or deletions in UMIs due to reverse transcription, PCR, and sequencing errors (Fig. 2b). We tested our method using total RNAs extracted from mouse testes isolated 17.5 days after birth. To assess the impact of the amount of starting materials on PCR duplicates, we prepared small RNA-seq libraries using a range of $39-5,000 \mathrm{ng}$ RNAs made from serial dilution. To test the effect of PCR cycles, we gradually increased the PCR cycles for each library with a two-cycle increment. The resulting UMI small RNA-seq libraries yielded high-quality sequencing data, comparable to those generated with the original non-UMI protocol (Additional file 1).

\section{Diverse UMIs capture all read species in RNA-seq and small RNA-seq}

As mentioned above, to accurately identify PCR duplicates using UMIs, it is critical that the number of distinct UMIs far exceeds the maximal number of starting molecules with identical sequences, such that these 
molecules have an infinitesimal probability of being ligated to adapters with the same UMI. Previous UMI methods were designed for sequencing single cells or an organism with a less complex transcriptome than mammals $[10,13]$. In particular, testis has a higher-complexity transcriptome than many other tissues such as muscle, liver, and even brain [29], demanding a large number of UMI combinations. Our UMI RNA-seq protocol theoretically provides $\sim 1$ million $\left(4^{10}\right)$ distinct combinations, and we asked whether this diversity far exceeded the maximal number of reads with identical sequences in our libraries. Indeed, the transcripts derived from the 299-bp 7S RNA 1 gene produce 19,271 identical reads mapping to the same genomic coordinate, all of which are attached to distinct UMI sequences, indicating that all of these reads were from different starting RNA molecules. In conclusion, our UMI RNA-seq protocol is more than sufficient to disambiguate biologically identical reads from PCR duplicates.

Our UMI small RNA-seq provides an even higher number of possible combinations with 18 nt UMIs-68.7 billion $\left(4^{18}\right)$-much larger than the number of reads currently produced by a sequencing run. The most abundant small RNA species in our datasets is a piRNA with 42,281 reads, far fewer than the number of UMI combinations our protocol provides. We conclude that the UMI lengths used in the RNA-seq and small RNA-seq protocols contain a sufficient UMI diversity for current and, most likely, future sequencing experiments.
Error-correction for UMIs only slightly improves PCR duplicate identification

To test whether UMIs could accurately identify PCR duplicates, we first evaluated their performance using simulated data. Assuming a library has sufficiently diverse UMI sequences, the simplest way to determine biologically identical reads is to look for reads with the same sequence but are tagged by different UMIs. This approach assumes that there is no error in the replication or reading of the UMI sequences, since such errors could render identical UMI sequences different and vice versa, causing misidentification of PCR duplicates. UMI errors could occur during PCR sequencing, and computationally correcting these errors has been shown to improve identification of PCR duplicates [14, 16, 30-32].

We designed a strategy for correcting UMI errors with the following considerations in mind. First, UMI errors are rare, with rates stipulated by the chemistry of PCR and sequencing $\left(\sim 10^{-5}\right.$ and $\sim 10^{-3}$ errors per position respectively) [33-36]. Second, when two sufficiently long UMIs (for example, 10 and $18 \mathrm{nt}$ in this study) that differ by just one base are connected to two reads with identical sequences, the probability that these are PCR duplicates of the same UMI with an error, albeit low $\left(p<10^{-3}\right)$ is still much higher than the probability that these are two distinct UMIs $\left(p=4^{-10}\right.$ for RNA-seq and $4^{-18}$ for small RNA-seq in this study). Adopting an error-correction method previously developed for RNA-seq [16], we built a UMI graph for each group of reads (Fig. 3a). For RNA-seq,

\begin{tabular}{|l} 
a \\
Intermediate ACGTGATGGA $\times 10$ \\
UMI required to \\
correct 2 errors
\end{tabular}


the reads that map to the same genomic position form a group. This approach does not work for small RNAs, because they often originate from multiple genomic loci. Thus, we simply defined a group of small RNA reads as those with identical sequences. In both the RNA-seq and small RNA-seq UMI graphs, a node denotes a unique UMI and further holds the number of reads with that UMI (Fig. 3a). For each pair of UMIs (say, UMI $a$ and UMI $b$ ) that differ by just one base (one edit distance apart), we connect their nodes if $n_{\mathrm{a}} \geq 2 \times n_{\mathrm{b}}-1$, where $n_{\mathrm{a}}$ and $n_{\mathrm{b}}$ represent read counts for the two UMIs. We require a twofold difference between $n_{\mathrm{a}}$ and $n_{\mathrm{b}}$, because as described above, the error rates for PCR and sequencing are low, and the twofold differences corresponds to the most extreme case whereby an error occurred during the first PCR cycle. However, a twofold difference is too stringent for pairs of UMIs with low read counts (e.g., 1 versus 2 ), for which the error predominantly arose from sequencing. We therefore added " -1 " to ensure that these UMIs could be connected. All connected UMIs are then assumed to originate from the most abundant UMIs in the graph. This scheme allows correction of two or more errors in UMIs, provided that the intermediate UMIs are observed (for example, the intermediate UMI with one error and UMI with two errors in Fig. 3a-b). One could relax the stringency of this method by adding direct connections between two nodes that differ in two or more positions.

The need for error-correction might depend on the experimental conditions, including the PCR amplification probability, PCR and sequencing error rates, UMI length, number of initial molecules, number of sequenced molecules, and number of PCR cycles. We performed computer simulations to investigate the effects of these seven experimental conditions on UMI error correction by systematically varying one variable at a time while holding the other six constant. Each round of simulation produced a known number of PCR duplicates and therefore, unlike experimental data, the true fraction of all reads corresponding to $\mathrm{PCR}$ duplicates can be determined in the simulated data. To assess the accuracy of PCR duplicate identification using UMIs, we calculated the difference between the number of reads after PCR duplicate removal ("estimate") and the true value ("truth") relative to the true value: (estimate - truth)/truth. This metric reflects the extent to which UMIs over- or underestimate the truth as a fraction of the true value. We started the simulation with 100 initial molecules. We then performed PCR by randomly assigning a probability to each molecule (tagged with an $18 \mathrm{nt}$ UMI) to be duplicated in each PCR cycle. The probability follows a uniform distribution between $m$ and 1 , where $m$ denotes minimum amplification probability (it can be any value between 0 and 1 and is set to 0.8 in the baseline condition). Minimum amplification probability can be interpreted as PCR efficiency, because the efficiency (average probability) that a molecule is doubled during each PCR cycle is $(1-m) / 2$. Ten cycles of PCR (PCR error rate set to $3 \times 10^{-5}$ ) [33-35] generated a pool of $61,000 \pm 1,000$ (mean \pm S.D.) molecules. To test the effect of sequencing depth, we randomly drew 100 molecules from the pool for sequencing (sequencing error rate set to $10^{-3}$ ) [36] (Fig. 4; Additional file 2: Figure S1). We call this set of parameters "baseline condition", and it forms the base line from which we systematically varied each parameter. For each condition, we performed 10,000 trials.

\section{No error correction, Error correction, Truth}
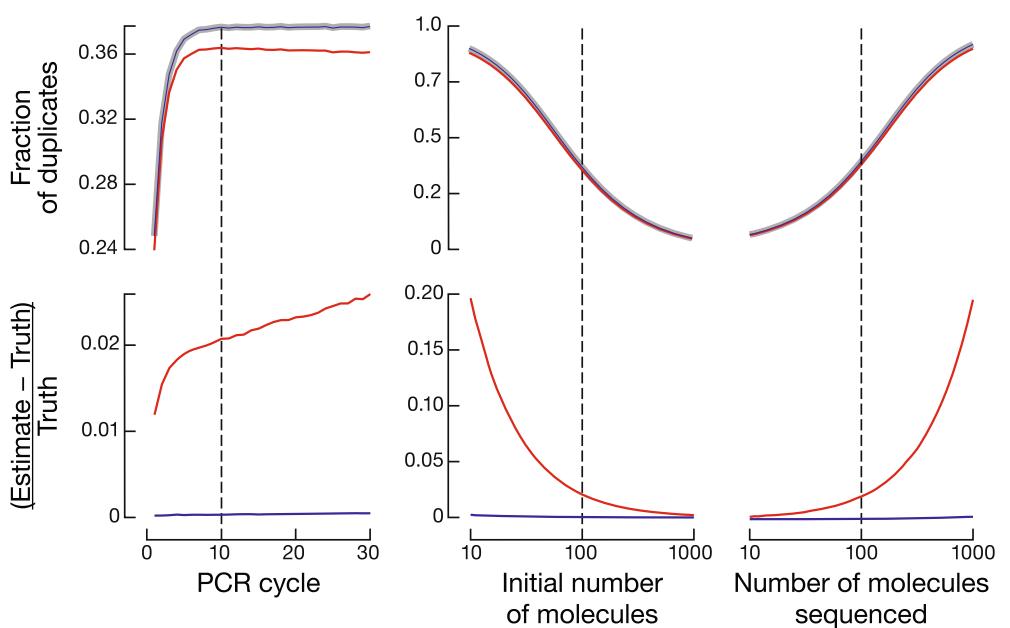

Fig. 4 Simulation of PCR duplicate removal with or without error correction for UMIs. One parameter (PCR cycle number, starting material, or sequencing depth) was varied with the other parameters kept constant. Upper plots show the fraction of duplicates, while lower plots show the accuracy of duplicate detection. Each dotted line indicates the value for this parameter used in other simulations 
We first assumed that there was no error in UMIs (Fig. 3a) and found that on average, (estimate - truth)/ truth $=2.10 \%$ across 10,000 trials under the baseline condition. Thus, without performing UMI error correction, we slightly overestimated the total number of biological molecules as an error in a UMI would artificially create an extra UMI, and in turn, we slightly underestimated the fraction of PCR duplicates (red vs gray lines in Fig. 4; Additional file 2: Figure S1). Next, we used the UMI graph approach described above (Fig. 3a, b) for correcting errors in UMIs, and the new average of (estimate - truth) $/$ truth $=0.0388 \%$. Even though correcting UMI errors consistently gives better (estimate truth)/truth than not correcting the errors, the absolute difference in the fractions of PCR duplicates between the two approaches is small (Fig. 4; Additional file 2: Figure S1). For example, under the baseline condition, the true fraction of duplicates was $37.8 \pm 3.2 \%$; without correcting UMI errors yielded $36.5 \pm 3.3 \%$, and correcting UMI errors gave $37.8 \pm 3.2 \%$.

Under some extreme conditions, correcting UMI errors yields substantially better results. For example, if we modify PCR error rate in the baseline condition from the default $3 \times 10^{-5}$ [33-35] to $10^{-3}$, correcting UMI errors still yields a fraction of duplicates $(37.2 \pm 3.2 \%)$ very close to the truth $(37.2 \pm 3.1 \%)$, while not correcting the errors underestimates the fraction of duplicates $(32.1 \pm 3.5 \%)$. In conclusion, error-correction for UMIs consistently, albeit slightly, improves PCR duplicate identification. Therefore, we performed error correction for all following analyses.

\section{Removing PCR duplicates without using UMIs is fundamentally flawed}

Does the practice of removing PCR duplicates without UMIs improve the quantification of both long and short transcripts and in particular, of small RNAs such as microRNAs or piRNAs, which collectively originate from a small portion of the genome? We compared PCR duplicate identification using UMIs together with mapping coordinates of the reads to the conventional approach of using coordinates alone.

When only mapping coordinates were used (RNA-seq data from eight mouse tissues) (Additional file 1), 16.444.5\% RNA-seq reads were determined to be PCR duplicates, whereas using UMI information in conjunction with coordinates identified only $1.89-10.67 \%$ as duplicates. That is, the majority of reads mapping to identical coordinates were in fact not PCR duplicates but rather from distinct starting molecules that should be counted for transcript abundance. The situation is even worse for small RNA-seq data, when only small RNA sequences were used, the majority $(56.0-76.8 \%)$ of reads were flagged as PCR duplicates and therefore excluded from analysis. In contrast, when UMI information was used together with the sequences of reads, just $1.05-13.6 \%$ of reads were determined to be duplicates. Thus, most of the identical reads in RNA-seq and small RNA-seq are biologically real and not PCR duplicates, consistent with the view that small RNAs, which tend to come from precisely the same small genomic regions, can easily be mistaken for PCR duplicates when UMI information is not used. Moreover, the assumption that common mapping coordinates indicate PCR duplicates becomes increasingly problematic as sequencing depth increases, because the chance of observing two identical reads that legitimately derive from different molecules before PCR also increases.

We further tested whether PCR duplicate removal using only mapping coordinates is appropriate for transcript quantification (Fig. 5a). The conventional method underestimated the abundance of 119 transcripts by 1.25 fold or more: removing PCR duplicates based only on coordinates is too aggressive. These 119 transcripts are significantly shorter (median length $=602 \mathrm{nt}$ ) and more highly expressed (median abundance $=200$ FPKM) than the other transcripts (median length $=1,620 \mathrm{nt}$; median abundance $=13.2$ FPKM; Wilcoxon rank sum test $p$ values $=2.22 \times 10^{-44}$ and $1.80 \times 10^{-59}$, respectively) (Fig. 5b). Thus, overestimation of PCR duplicates without UMIs reflects (1) a higher tendency of short transcripts to produce identical fragments due to more limited possibilities in fragmentation, and (2) a higher tendency of highly expressed genes to produce identical fragments. We conclude that removing PCR duplicates in RNA-seq data solely by mapping coordinates introduces substantial bias for short or highly expressed genes and that UMIs allow more accurate quantification of PCR duplicates and transcript abundance.

\section{UMls improve data reproducibility}

One metric for evaluating the quality of experimental data is the reproducibility between technical replicates. We evaluated how UMIs affect the reproducibility of transcript quantification using five libraries generated using the same sample of total mouse testis RNA, but with gradually decreasing amounts of starting RNA and correspondingly increasing numbers of PCR cycles: $4 \mu \mathrm{g}$ (8 PCR cycles), $2 \mu \mathrm{g}$ (9 PCR cycles), $1 \mu \mathrm{g}$ (10 PCR cycles), 500 ng (11 PCR cycles), 125 ng (13 PCR cycles) (Additional file 1). We then analyzed the data sets treating PCR duplicates using one of three approaches: (1) no PCR duplicates were removed; (2) PCR duplicates were removed using the conventional approach of identical genomic locations; and (3) PCR duplicates were removed using UMIs together with mapping coordinates. We compared the three approaches by calculating coefficients of variation $(\mathrm{CV}=$ S.D./mean) for transcript 
a

b

Abundance underestimated $\geq 1.25 \times$ using mapping coordinates only
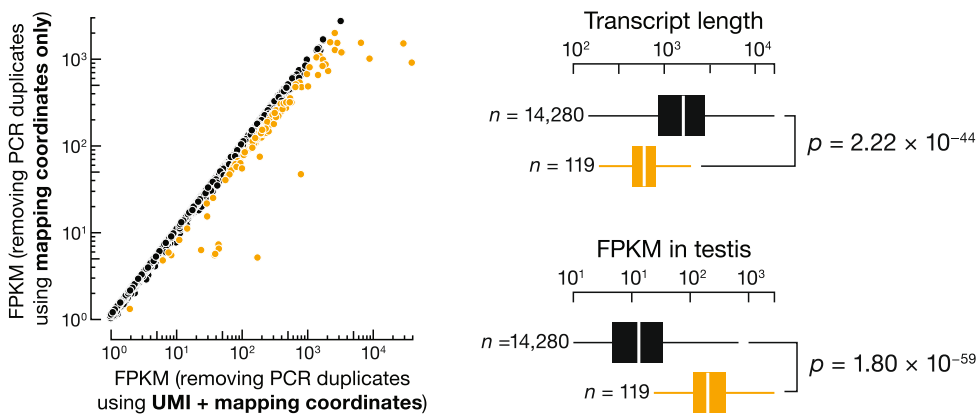

C

UMI RNA-seq

UMI small RNA-seq

PCR duplicates not removed, removed using

mapping coordinates only or UMI + mapping coordinates
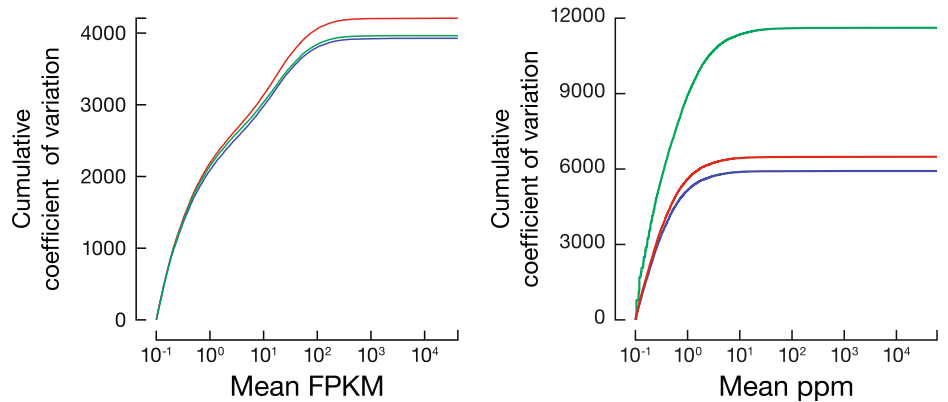

Fig. 5 a Transcript abundance (FPKM) calculated by removing PCR duplicates using only mapping coordinates compared to using mapping coordinates and UMIs. $\mathbf{b}$ Using only mapping coordinates significantly biases against abundant and short genes. Outliers omitted. Wilcoxon rank sum test; $n$, number of genes in each group. c Relationship between cumulative coefficient of variation and transcript abundance

abundance across the five RNA-seq libraries. Compared to removing no duplicates, removing duplicates according to their mapping coordinates decreased the total CV by $5.80 \%$ (from 4,210 to 3,960 ), while using UMIs with mapping coordinates decreased the total CV by $6.67 \%$ (from 4,210 to 3,930) (Fig. 5c). For example, when two RNA-seq libraries (125 ng with 12 PCR cycles and $1 \mu \mathrm{g}$ with 10 PCR cycles) were compared, the number of transcripts whose abundance differed by $\geq 25 \%$ decreased when duplicates were removed (1,880 without duplicate removal, 1,503 removing duplicates by genomic coordinates, and 1,415 removing duplicates using UMIs). We conclude that removing PCR duplicates in RNA-seq data using mapping coordinates alone improves the overall precision of transcript quantification for all genes, and removing PCR duplicates by combining coordinates and UMIs further improves the precision, albeit slightly.

Next, we evaluated the performance of these three approaches for a series of small RNA-seq libraries (starting material 39-5,000 ng). Compared to removing no duplicates, using UMIs to remove duplicates decreased the total CV by $8.72 \%$ (Fig. 5c). Surprisingly, removing duplicates according to their mapping coordinates alone increased CV by $79.1 \%$ (from 6,490 to 11,620 ) (Fig. 5c). For example, between two small RNA-seq libraries in this series, one generated from $150 \mathrm{ng}$ and the other from $1 \mu \mathrm{g}$ of the same total RNA sample, genomic loci (piRNA genes and GENCODE-annotated genes) whose small RNA abundance differed by $\geq 25 \%$ decreased $8.30 \%$ when duplicates were removed using UMIs (from 2,613 to 2,396 genes). In contrast, when duplicates were removed using solely mapping coordinates, the number of such irreproducible genes increased by $159 \%(6,762$ genes). These results show that removing PCR duplicates with UMIs leads to more consistent quantification across libraries, whereas removing duplicates without UMIs is overly aggressive and decreases the reproducibility of small RNA-seq experiments.

\section{PCR cycles alone do not determine the frequency of PCR duplicates}

One might think that the number of PCR cycles used to amplify the initial cDNA is the major cause of PCR duplicates in sequencing libraries [37]. We sought to test this and to identify other experimental contributing factors. As described above, we performed computer simulations to 
test the impact of UMI error correction on PCR duplicate detection. We considered seven parameters that could impact the level of PCR duplicates during an RNA-seq or small RNA-seq experiment. Assuming that we have performed UMI error correction, we now examine in detail these seven parameters for their impact on the level of PCR duplicates.

Four of the parameters-PCR amplification efficiency, PCR error rate, sequencing error rate, and UMI lengthare specified by the experimental reagents and sequencing platform and typically not adjusted from experiment to experiment. Our simulation results indicate that varying the sequencing error rate, the PCR error rate, or the UMI length around their default values in the baseline condition (i.e., within the ranges stipulated by experimental settings) did not have a significant effect on the fraction of PCR duplicates (the blue line is flat around the dashed vertical line in Additional file 2: Figure S1A-C, top panels). In comparison, PCR efficiency had a measurable effect (the blue line in the top panel of Additional file 2: Figure S1D reveals a negative correlation with PCR efficiency). This is because that at lower PCR efficiency, some molecules are less likely to be amplified and become underrepresented, causing a decrease in library complexity and correspondingly higher fractions of PCR duplicates.

The other three parameters-the number of initial molecules, the number of molecules sequenced (i.e., sequencing depth), and the number of PCR cycles-are often adjusted to meet specific experimental conditions. Our simulations revealed that a change in PCR cycle number alone only minimally affected the fraction of PCR duplicates (the blue line in the top-left panel of Fig. 4 is nearly flat around the dashed vertical line), because the starting molecules of the original pool are proportionally propagated to the final library [38]. In contrast, decreasing the number of initial molecules or increasing the number of molecules sequenced sharply raised the frequency of PCR duplicates (Fig. 4, two top-right panels).

We further tested these findings using experimental datasets. We first analyzed a set of five UMI RNA-seq libraries made with gradually decreasing amounts of starting RNA and correspondingly increasing numbers of PCR cycles: $4 \mu \mathrm{g}$ (8 cycles), $2 \mu \mathrm{g}$ (9 cycles), $1 \mu \mathrm{g}$ (10 cycles), $500 \mathrm{ng}$ (11 cycles), $125 \mathrm{ng}$ (13 cycles) (Additional file 1). We observed that less starting RNA and correspondingly more PCR amplification resulted in higher fractions of PCR duplicates (Fig. 6a). For example, the $125 \mathrm{ng}$, 13-cycle library yielded $10.7 \%$ (median over 43,432 genes) PCR duplicates, while the $4 \mu \mathrm{g}$, 8-cycle library made by the same procedure contained only $1.79 \%$ PCR duplicates. Similarly, analysis of UMI small RNA-seq libraries generated from $39 \mathrm{ng}$ ( 30 cycles) to $5 \mu \mathrm{g}$ ( 16 cycles) total RNA (Additional file 1) revealed that starting with less RNA caused higher fractions of PCR duplicates (Fig. 6a).

Simulations argue that the increase in PCR duplicates is not a consequence of greater PCR amplification but rather is caused by the use of lower starting material. To test this idea, we analyzed a second set of nine UMI small RNA-seq libraries, all generated from $5 \mu \mathrm{g}$ total RNA from the same mouse testis, but amplified using 14 to 30 PCR cycles (Additional file 1). Consistent with the simulations, these libraries did not show a discernable trend between fraction of PCR duplicates and the number of PCR cycles (Fig. 6b). Thus, the higher fraction of PCR duplicates observed in libraries made from low amounts of RNA followed by high PCR cycle numbers more likely reflects the reduced complexity of the starting pool, rather than the increased number of PCR cycles. Together, our simulated and experimental data demonstrate that less starting RNA or higher sequencing depth, but not more PCR cycles per se, accounts for the frequency of PCR duplicates. Nonetheless, our recommendation remains to optimize PCR cycle numbers during
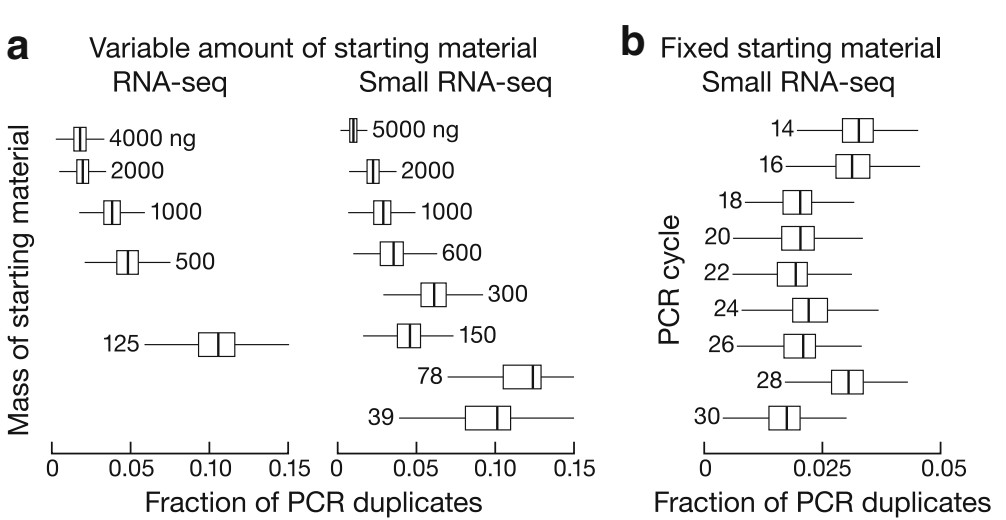

Fig. 6 Fraction of PCR duplicates across genes for (a) a series of UMI RNA-seq and small RNA-seq libraries made with different amount of starting materials, and (b) a series of UMI small RNA-seq libraries all made with 5 Mg of total mouse testis RNA and with an increasing number of PCR cycles 
library preparation because several other artifacts are known to be associated with excessive PCR amplification, such as decreasing amplification efficiency as PCR reaction proceeds [39], biased amplification of sequences with different GC content [40], or chimeric PCR products due to cross hybridization [41, 42]. Moreover, when the starting material has low complexity, as for single-cell sequencing, excessive PCR amplification is likely to compound all such problems.

\section{Discussion}

The two most widely used computational tools for PCR duplicate removal, Picard MarkDuplicates (http://broadinstitute.github.io/picard/) and SAMtools rmdup [3] rely only on the mapping coordinates of sequencing reads. Our data suggest that most identical reads reflect biological reality. Thus, removing PCR duplicate reads using only mapping coordinates erroneously eliminates many usable reads, particularly those produced from short transcripts and small RNAs. This is consistent with an earlier study that compared RNA-seq data generated using three different library preparation methods (Smart-Seq, TruSeq and UMI-seq) [43] and reported that removing PCR duplicates without UMIs introduced bias into RNA-seq data. There are also computational methods that assess PCR duplicate rates without UMIs, e.g., using reads mapping to heterozygous variants [44] or gene expression levels [45], but they only estimate duplicate frequencies and cannot accurately identify duplicate reads. Our approach relies on UMIs and can accurately identify all PCR duplicates, regardless of gene expression and genomic variants.

The eight mouse tissues we analyzed span a range of transcriptome complexity: previous analyses showed that the mouse testis transcriptome contains $\sim 18,700$ autosomal protein-coding transcripts, $\sim 8,600$ non-coding RNAs, and $\sim 31.7 \mathrm{Mb}$ of intergenic RNA, while the liver transcriptome contains only $\sim 15,500$ autosomal proteincoding transcripts, $\sim 1,000$ non-coding RNAs, and $\sim$ 7.2 $\mathrm{Mb}$ of intergenic RNA [29]. Among the eight mouse tissues we tested, removing duplicate reads based on only mapping coordinates eliminates many biologically meaningful reads even when the libraries were made using ample starting RNA and optimal experimental conditions. Given the anti-correlation between RNA complexity and PCR duplicate occurrence, UMIs will improve the accuracy of comparing long or small RNA abundance across different tissues or cell types. Short RNAs, such as miRNAs and piRNAs, as well as highly abundant transcripts are particularly susceptible to underestimation by the conventional mapping coordinate method of PCR duplicate removal.

We tested the importance of a key aspect of data processing required for error correction using UMIs and showed that under typical experimental conditions for bulk sequencing (Fig. 4, dotted lines; Additional file 2: Figure S1), correcting or not correcting errors in the UMI sequences has little absolute effect on PCR duplicate quantification. However, sequencing libraries made from a small number of cells or from a small amount of tissue or RNA, have become increasingly common [46], and they are more severely affected by PCR duplicates. Single-cell sequencing poses three specific challenges for PCR duplicate removal. First, it uses a limited amount of starting RNA, causing low library complexity. Second, the ongoing discovery of new species of non-coding RNAs, many poorly understood, increases the number of species being measured, requiring longer UMIs. Finally, the increasingly high sequencing depth provided by advances in technology increases both the number of species that can be detected and the background noise. Together, these three factors make PCR duplicate measurement without UMI error correction especially problematic for single-cell sequencing. Indeed, a number of protocols have been developed to incorporate UMIs for single-cell sequencing [47]. We compile all UMI methods known to us in Additional file 3, including bulk and single-cell, RNA-seq and small RNA-seq protocols.

Compared to other UMI protocols (Additional file 3), our method has several novel features. First, our UMI RNA-seq protocol fragments the RNA before random hexamer priming of cDNA synthesis. It captures both poly(A) + and poly(A) - RNA while limiting 3 '-end bias. In comparison, template-switching cDNA synthesis is commonly used in single-cell sequencing protocols; it captures full length cDNAs more efficiently but is also prone to significant 3 '-end bias and loss of poly(A) - RNA species due to oligo(dT) priming [14, 48, 49]. Second, our UMI RNA-seq protocol has yielded high-quality data from 125 ng starting material and our UMI small RNA-seq protocol from $39 \mathrm{ng}$. Our protocols do not require pre-amplification and perform fragmentation prior to cDNA synthesis. Consequently, these methods capture all PCR duplicates. Third, our protocol can accommodate a large number of UMIs: random sequences with $5 \times 2 \mathrm{nt}$ for RNA-seq and $9 \times 2$ nt for small RNA-seq. Thus, our protocol can be used to study RNA with high sequence diversity: e.g. piRNA in the germline. In our hands long stretches of random nucleotides interferes with library construction and sequencing, whereas our use and placement of UMI locators improves sequencing quality. With the increasing use of small amounts of starting materials, our protocol complements other approaches (Additional file 3).

Our UMI approach builds on well-established protocols, requiring few changes in the procedures and little additional cost. We expect UMI analysis to be particularly useful when sequencing RNAs derived from a 
limited number of genomic loci, such as CaptureSeq [50] and CAGE-seq [51]. Our approach can theoretically be adapted to any sequencing technique using synthetic oligonucleotide adapters. For example, chromatin immunoprecipitation sequencing (ChIP-seq) and its alternative CUT\&RUN survey the genomic regions bound by proteins of interest $[52,53]$. The CUT\&RUN method uses a nuclease to achieve more precise chromatin cleavage than the conventional ChIP-seq procedure, which utilizes sonication to randomly shear the DNA. Consequently, CUT\&RUN increases the likelihood of identical but meaningful reads. By their nature, protein-bound fragments also map to a smaller portion of genomic positions than RNA-seq reads. UMIs can improve discovery of protein binding sites by minimizing noise. Similarly, degradome sequencing profiles the $5^{\prime}$ ends of 3' cleaved RNA products [54]; incorporating UMIs will enable precise quantification of cleaved RNA abundance.

\section{Conclusions}

We described experimental protocols and computational methods that, by incorporating UMIs into standard procedures, allow accurate PCR duplicate removal from RNA-seq and small RNA-seq data. Our approach increases reproducibility and decreases noise in sequencing libraries generated using a broad range of starting RNA amount and number of PCR cycles, enabling accurate quantification of the abundance of both long and short RNAs.

\section{Methods}

\section{Animals}

C57BL/6 J mice were sacrificed using $\mathrm{CO}^{2}$ and cervical dislocation and tissues were collected from mice maintained and used according to guidelines approved by the Institutional Animal Care and Use Committee of the University of Massachusetts Medical School (A-2222-17).

\section{Ribosomal RNA depletion for RNA-seq}

Total RNA was extracted from tissues using the mirVana kit (ThermoFisher Scientific, Waltham, MA, USA) following manufacturer's instructions. The ribosomal RNA depletion method was adapted from previously published protocols for human samples [55, 56]. One hundred and eighty-six 50 nt-long DNA oligonucleotides complementary to the entire sequences of mouse $18 \mathrm{~S}$, 28S, 5S, and 5.8S rRNAs, and mitochondria 16S rRNA and $16 \mathrm{~S}$ rRNA precursor were used at $0.5 \mu \mathrm{M}$ (f.c.) for each oligonucleotide. Total mouse testis RNA was isolated with mirVana and incubated with $1 \mu \mathrm{L}$ of the DNA oligonucleotide mixture per $1 \mu \mathrm{g}$, and rRNA oligonucleotide hybridization buffer $(100 \mathrm{mM}$ Tris- $\mathrm{Cl} \mathrm{pH}$ 7.4, $200 \mathrm{mM} \mathrm{NaCl}$ ) was added to make up to $10 \mu \mathrm{L}$. Oligonucleotide hybridization was carried out by heating the sample at $95{ }^{\circ} \mathrm{C}$ for $3 \mathrm{~min}$, then slowly cooling it down $\left(-0.1{ }^{\circ} \mathrm{C} /\right.$ second $)$ to $22{ }^{\circ} \mathrm{C}$ in a thermocycler. The reaction was further incubated at $22{ }^{\circ} \mathrm{C}$ for $5 \mathrm{~min}$ before being placed on ice. Thermostable RNase $\mathrm{H}$ (Lucigen, Middleton, MA) was added (5 U/ $\mu$ g total RNA), and the reaction adjusted to $50 \mathrm{mM}$ Tris- $\mathrm{Cl} \mathrm{pH} \mathrm{7.4,} 100 \mathrm{mM}$ $\mathrm{NaCl}$, and $20 \mathrm{mM} \mathrm{MgCl} 2$. and incubated at $45{ }^{\circ} \mathrm{C}$ for $30 \mathrm{~min}$. After DNase treatment with Turbo DNase ( $1 \mu \mathrm{L} / \mu \mathrm{g}$ total RNA) according to the manufacturer's instructions, the rRNA-depleted RNA was purified using RNA Clean \& Concentrator-5 (Zymo Research, Irvine, CA, USA).

\section{RNA-seq library construction}

RNA-seq library generation was similar to previously published [17], except for the use of a more effective rRNA deletion method (above) and the UMI adapters. Briefly, total RNA was randomly fragmented using heat $\left(94{ }^{\circ} \mathrm{C}\right.$ for $5 \mathrm{~min}$ ) and $\mathrm{Mg}^{2+}$. First strand cDNA was generated using ribosomal-depleted, fragmented total RNA with Superscript III (Invitrogen, Carlsbad, CA, USA). cDNA then underwent dUTP incorporation, end repair, and A-tailing. Resulting cDNA was incubated with a mixture of three sets of UMI-containing adapters, each carrying a distinct consensus sequence as described in the results for adapter ligation (Fig. 1b-c). The ligated cDNA was treated with UDG (New England Biolabs, Ipswich, MA, USA) and amplified by PCR. Length distribution and quality of the resulting library was analyzed by Agilent 2100 Bioanalyzer (Agilent Genomics, Santa Clara, MA, USA). The libraries were quantified using KAPA library quantification kit (KAPA Biosystems, Wilmington, MA, USA) and sequenced using NextSeq 500 (Illumina, San Diego, CA, USA) paired-end sequencing.

\section{Small RNA-seq library construction}

Small RNA-seq library preparation was similar to previously described $[24,25]$ with updated modifications. Briefly, 18-35 nt mouse small RNA was purified from a 15\% denaturing urea-polyacrylamide gel (National Diagnostics, Atlanta, GA, USA). Small RNA was first ligated to 3' DNA adapters with adenylated $5^{\prime}$ and dideoxycytosine-blocked $3^{\prime}$ ends in $50 \mathrm{mM}$ Tris- $\mathrm{HCl}(\mathrm{pH} 7.5), 10 \mathrm{mM} \mathrm{MgCl}$, $10 \mathrm{mM}$ DTT, and 50\% PEG 8000 (New England Biolabs, Ipswich, MA, USA) with T4 Rnl2tr K227Q (homemade) at $25{ }^{\circ} \mathrm{C}$ for $16 \mathrm{~h}$. The $3^{\prime}$ adapter contained UMIs in $3 \mathrm{nt}$-blocks of random nucleotides separated by pre-defined 3 nt consensus sequences (NNN-GTC-NNN-TAG-NNN, Fig. 2b). The 54-71 nt (18-35 nt small RNA + 36 nt 3' UMI adapter) $3{ }^{\prime}$ ligated product was purified from a $10 \%$ denaturing urea-polyacrylamide gel. The 3' ligated product was then ligated to a mixed pool of equimolar amount of 5' RNA adapters containing UMIs in 3 nt-blocks of random nucleotides and one of the two distinct consensus sequence sets (NNN-CGA-NNN-UAC-NNN 
and NNN-AUC-NNN-AGU-NNN) in $50 \mathrm{mM}$ Tris-HCl (pH 7.8), $10 \mathrm{mM} \mathrm{MgCl}$, $10 \mathrm{mM}$ DTT, $1 \mathrm{mM}$ ATP with T4 RNA ligase (Ambion, Foster City, CA, USA) at $25{ }^{\circ} \mathrm{C}$ for $2 \mathrm{~h}$. The ligated product was precipitated with ethanol, and cDNA synthesis was performed using AMV reverse transcriptase (New England Biolabs, Ipswich, MA, USA). cDNA was PCR-amplified with a common forward primer (5'-AAT GAT ACG GCG ACC ACC GAC AGG TTC AGA GTT CTA CAG TCC GA-3') and a reverse primer containing 6 nt Illumina multiplexing barcode $\left(5^{\prime}-\right.$ CAA GCA GAA GAC GGC ATA CGA GAT NNN NNN GTG ACT GGA GTT CCT TGG CAC CCG AGA ATT CCA-3') using AccuPrime $P f x$ DNA polymerase (ThermoFisher, Waltham, MA, USA). Finally, the PCR product was purified from a $2 \%$ Certified Ultra Low Range agarose gel (Bio-Rad Laboratories, Hercules, CA, USA). Length distribution and quality of the resulting libraries was analyzed by Agilent 2100 Bioanalyzer (Agilent Technologies, Santa Clara, CA, USA). The libraries were quantified using the KAPA library quantification kit (KAPA Biosystems, Wilmington, MA, USA) and sequenced using NextSeq 500 single-end sequencing (Illumina, San Diego, CA, USA).

\section{Bioinformatics}

Simulation procedure was performed according to [16]. Briefly, we simulated 7 parameters: PCR amplification probability, PCR and sequencing error rates, UMI length, number of initial molecules, number of sequenced molecules, and PCR cycle numbers, by varying one parameter and keeping other parameters constant. For each combination of the 7 parameters, we performed 10,000 replicates. UMI error correction was implemented as described in [16], except that we used read sequences instead of genomic coordinates when determining PCR duplicates for small RNA-seq. We used NetworkX [57] for graph-related algorithms, and pysam (https://github.com/ pysam-developers/pysam) for handling SAM/BAM files. Reads were mapped to the mouse mm10 genome as described in [58]. For each UMI RNA-seq read, the UMI locator was required to be a perfect match of GGG, TCA, or ATC. For each UMI small RNA-seq read, the 5' UMI locator was required to match NNNCGANNNTACNNN or NNNATCNNNAGTNNN and the 3' UMI locator was required to match NNNGTCNNNTAGNNN, allowing 1 error across all of the non-N positions. Reads not meeting these requirements were discarded. UMIs in RNA-seq data were identified using umitools reformat_fastq. Subsequently, PCR duplicates were marked using umitools mark_duplicates. UMIs in small RNA-seq were identified and removed using umitools reformat_sra_fastq. When reads were analyzed without UMIs, PCR duplicates were identified using MarkDuplicates from Picard (https://github.com/broadinstitute/picard).

\section{Additional files}

Additional file 1: Mapping and UMI statistics of (A) RNA-seq and (B) small RNA-seq data generated in this study. (XLSX $112 \mathrm{~kb}$ )

Additional file 2: Figure S1. Accuracy and fraction of duplicates for simulated data varying (A) sequencing error rate, (B) UMl length, $(C)$ PCR error rate, or (D) minimum amplification probability. Each dotted line indicates the value for this parameter used in other simulations. (PDF $868 \mathrm{~kb}$ )

Additional file 3: UMI method comparison. (XLSX $11 \mathrm{~kb}$ )

\section{Abbreviations}

CV: Coefficient of variation; miRNA: microRNA; PCR: Polymerase chain reaction; piRNA: PIWI-interacting RNA; UMI: Unique molecular identifier

\section{Acknowledgements}

We thank members of the Weng and Zamore laboratories for helpful discussions and comments on the manuscript.

\section{Funding}

This work was supported in part by National Institutes of Health grants P01HD078253 and R37GM062862 to PDZ and HD078253 to ZW. The funding agency had no role in the design of the study and collection, analysis, and interpretation of data and in writing the manuscript.

\section{Availability of data and materials}

The tools developed for handling UMIs in our RNA-seq and small RNA-seq data can be found at https://github.com/weng-lab/umitools, and via PyPI (package: umitools). RNA-seq and small RNA-seq data have been deposited in the NCBI SRA under the accession number PRJNA416930.

\section{Authors' contributions}

All authors have read and approved the final version of the manuscript. YF, Bioinformatics analysis, Writing; PHW, Protocol design, RNA-seq, small RNAseq, Writing; TB, Small RNA-seq; ZW, Project design and management, Writing; PDZ, Project conception, design and management, Writing.

\section{Ethics approval}

The Institutional Animal Care and Use Committee of the University of Massachusetts Medical School (A-2222-17) provided official ethics board approval for this study. Tissues used in this study were collected from C57BL/6 J mice (Jackson Laboratory, stock number 664) maintained and used according to the approved protocols.

\section{Consent for publication}

Not applicable.

\section{Competing interests}

The authors declare that they have no competing interests.

\section{Author details \\ ${ }^{1}$ Bioinformatics Program, Boston University, 44 Cummington Mall, Boston, MA 02215, USA. ²Program in Bioinformatics and Integrative Biology, University of Massachusetts Medical School, 368 Plantation Street, Worcester, MA 01605, USA. ${ }^{3}$ RNA Therapeutics Institute and Howard Hughes Medical Institute, University of Massachusetts Medical School, 368 Plantation Street, Worcester, MA 01605, USA. ${ }^{4}$ Department of Biochemistry and Molecular Pharmacology, University of Massachusetts Medical School, 368 Plantation Street, Worcester, MA 01605, USA.}

Received: 9 March 2018 Accepted: 8 July 2018

Published online: 13 July 2018

\section{References}

1. Cha RS, Thilly WG. Specificity, efficiency, and fidelity of PCR. PCR Methods Appl. 1993;3:S18-29.

2. Dohm JC, Lottaz C, Borodina T, Himmelbauer H. Substantial biases in ultrashort read data sets from high-throughput DNA sequencing. Nucleic Acids Res. 2008;36:e105. 
3. Li H, Handsaker B, Wysoker A, Fennell T, Ruan J, Homer N, Marth G, Abecasis G, Durbin R, 1000 GPDPS. The Sequence Alignment/Map format and SAMtools. Bioinformatics. 2009;25:2078-9.

4. Brennecke J, Aravin AA, Stark A, Dus M, Kellis M, Sachidanandam R, Hannon GJ. Discrete small RNA-generating loci as master regulators of transposon activity in Drosophila. Cell. 2007;128:1089-103.

5. Aravin A, Gaidatzis D, Pfeffer $S$, Lagos-Quintana M, Landgraf P, lovino N, Morris P, Brownstein MJ, Kuramochi-Miyagawa S, Nakano T, Chien M, Russo JJ, Ju J, Sheridan R, Sander C, Zavolan M, Tuschl T. A novel class of small RNAs bind to MILI protein in mouse testes. Nature. 2006:442:203-7.

6. Girard A, Sachidanandam R, Hannon GJ, Carmell MA. A germline-specific class of small RNAs binds mammalian Piwi proteins. Nature. 2006;442:199-202.

7. Li XZ, Roy CK, Dong X, Bolcun-Filas E, Wang J, Han BW, Xu J, Moore MJ, Schimenti JC, Weng Z, Zamore PD. An ancient transcription factor initiates the burst of piRNA production during early meiosis in mouse testes. Mol Cell. 2013;50:67-81.

8. Kivioja T, Vähärautio A, Karlsson K, Bonke M, Enge M, Linnarsson S, Taipale J. Counting absolute numbers of molecules using unique molecular identifiers. Nat Methods. 2011;9:72-4.

9. Fu GK, Hu J, Wang PH, Fodor SP. Counting individual DNA molecules by the stochastic attachment of diverse labels. Proc Natl Acad Sci U S A. 2011;108:9026-31.

10. Fu GK, Wilhelmy J, Stern D, Fan HC, Fodor SP. Digital encoding of cellular mRNAs enabling precise and absolute gene expression measurement by single-molecule counting. Anal Chem. 2014;86:2867-70.

11. Fu GK, Xu W, Wilhelmy J, Mindrinos MN, Davis RW, Xiao W, Fodor SP. Molecular indexing enables quantitative targeted RNA sequencing and reveals poor efficiencies in standard library preparations. Proc Natl Acad Sci U S A. 2014;111:1891-6.

12. Collins JE, Wali N, Sealy IM, Morris JA, White RJ, Leonard SR, Jackson DK, Jones MC, Smerdon NC, Zamora J, Dooley CM, Carruthers SN, Barrett JC, Stemple DL, Busch-Nentwich EM. High-throughput and quantitative genome-wide messenger RNA sequencing for molecular phenotyping. BMC Genomics. 2015;16:578

13. Shiroguchi K, Jia TZ, Sims PA, Xie XS. Digital RNA sequencing minimizes sequence-dependent bias and amplification noise with optimized singlemolecule barcodes. Proc Natl Acad Sci U S A. 2012;109:1347-52.

14. Islam S, Zeisel A, Joost S, La Manno G, Zajac P, Kasper M, Lönnerberg P, Linnarsson S. Quantitative single-cell RNA-seq with unique molecular identifiers. Nat Methods. 2014;11:163-6.

15. Girardot C, Scholtalbers J, Sauer S, Su SY, Furlong EE. Je, a versatile suite to handle multiplexed NGS libraries with unique molecular identifiers. BMC Bioinformatics. 2016;17:419.

16. Smith T, Heger A, Sudbery I. UMI-tools: modeling sequencing errors in Unique Molecular Identifiers to improve quantification accuracy. Genome Res. 2017;27:491-9.

17. Zhang Z, Theurkauf WE, Weng Z, Zamore PD. Strand-specific libraries for high throughput RNA sequencing (RNA-Seq) prepared without poly(A) selection. Silence. 2012;3:9.

18. Mohn F, Sienski G, Handler D, Brennecke J. The rhino-deadlock-cutoff complex licenses noncanonical transcription of dual-strand piRNA clusters in drosophila. Cell. 2014;157:1364-79.

19. Zhang Z, Wang J, Schultz N, Zhang F, Parhad SS, Tu S, Vreven T, Zamore PD, Weng Z, Theurkauf WE. The HP1 homolog rhino anchors a nuclear complex that suppresses piRNA precursor splicing. Cell. 2014;157:1353-63.

20. Hayashi R, Handler D, Ish-Horowicz D, Brennecke J. The exon junction complex is required for definition and excision of neighboring introns in Drosophila. Genes Dev. 2014;28:1772-85.

21. Mitra A, Skrzypczak M, Ginalski K, Rowicka M. Strategies for achieving high sequencing accuracy for low diversity samples and avoiding sample bleeding using illumina platform. PLoS One. 2015;10:e0120520.

22. Illumina: Illumina: Low-Diversity Sequencing on the Illumina HiSeq ${ }^{\circledR}$ Platform. Technical Note: DNA Sequencing 2014,

23. Illumina: Illumina: NextSeq ${ }^{\circledast} 500$ System Guide. Technical Note: DNA Sequencing 2016,

24. Zhang Z, Xu J, Koppetsch BS, Wang J, Tipping C, Ma S, Weng Z, Theurkauf WE, Zamore PD. Heterotypic piRNA Ping-Pong requires qin, a protein with both E3 ligase and Tudor domains. Mol Cell. 2011;44:572-84.

25. Li C, Vagin W, Lee S, Xu J, Ma S, Xi H, Seitz H, Horwich MD, Syrzycka M, Honda BM, Kittler EL, Zapp ML, Klattenhoff C, Schulz N, Theurkauf WE, Weng Z, Zamore PD. Collapse of germline piRNAs in the absence of Argonaute3 reveals somatic piRNAs in flies. Cell. 2009;137:509-21.
26. Aravin AA, Sachidanandam R, Girard A, Fejes-Toth K, Hannon GJ. Developmentally regulated piRNA clusters implicate MILI in transposon control. Science. 2007;316:744-7.

27. Houwing S, Kamminga LM, Berezikov E, Cronembold D, Girard A, van den Elst H, Filippov DV, Blaser H, Raz E, Moens CB, Plasterk RH, Hannon GJ, Draper BW, Ketting RF. A role for Piwi and piRNAs in germ cell maintenance and transposon silencing in Zebrafish. Cell. 2007;129:69-82.

28. Yue F, Cheng Y, Breschi A, Vierstra J, Wu W, Ryba T, Sandstrom R, Ma Z, Davis C, Pope BD, Shen Y, Pervouchine DD, Djebali S, Thurman RE, Kaul R, Rynes E, Kirilusha A, Marinov GK, Williams BA, Trout D, Amrhein H, FisherAylor K, Antoshechkin I, DeSalvo G, See LH, Fastuca M, Drenkow J, Zaleski C, Dobin A, Prieto P, Lagarde J, Bussotti G, Tanzer A, Denas O, Li K, Bender MA, Zhang M, Byron R, Groudine MT, McCleary D, Pham L, Ye Z, Kuan S, Edsall L, Wu YC, Rasmussen MD, Bansal MS, Kellis M, Keller CA, Morrissey CS, Mishra T, Jain D, Dogan N, Harris RS, Cayting P, Kawli T, Boyle AP, Euskirchen G, Kundaje A, Lin S, Lin Y, Jansen C, Malladi VS, Cline MS, Erickson DT, Kirkup VM, Learned K, Sloan CA, Rosenbloom KR, Lacerda de Sousa B, Beal K, Pignatelli M, Flicek P, Lian J, Kahveci T, Lee D, Kent WJ, Ramalho Santos M, Herrero J, Notredame C, Johnson A, Vong S, Lee K, Bates D, Neri F, Diegel M, Canfield T, Sabo PJ, Wilken MS, Reh TA, Giste E, Shafer A, Kutyavin T, Haugen E, Dunn D, Reynolds AP, Neph S, Humbert R, Hansen RS, De Bruijn M, Selleri L, Rudensky A, Josefowicz S, Samstein R, Eichler EE, Orkin SH, Levasseur D, Papayannopoulou T, Chang KH, Skoultchi A, Gosh S, Disteche C, Treuting P, Wang Y, Weiss MJ, Blobel GA, Cao X, Zhong S, Wang T, Good PJ, Lowdon RF, Adams LB, Zhou XQ, Pazin MJ, Feingold EA, Wold B, Taylor J, Mortazavi A, Weissman SM, Stamatoyannopoulos JA, Snyder MP, Guigo R, Gingeras TR, Gilbert DM, Hardison RC, Beer MA, Ren B, Mouse ENCODEC. A comparative encyclopedia of DNA elements in the mouse genome. Nature. 2014;515:355-64.

29. Soumillon $M$, Necsulea $A$, Weier $M$, Brawand $D$, Zhang $X, G u$, Barthès $P$, Kokkinaki M, Nef S, Gnirke A, Dym M, de Massy B, Mikkelsen TS, Kaessmann $\mathrm{H}$. Cellular source and mechanisms of high transcriptome complexity in the mammalian testis. Cell Rep. 2013;3:2179-90.

30. Bose S, Wan Z, Carr A, Rizvi AH, Vieira G, Pe'er D, Sims PA. Scalable microfluidics for single-cell RNA printing and sequencing. Genome Biol. 2015;16:120.

31. Macosko EZ, Basu A, Satija R, Nemesh J, Shekhar K, Goldman M, Tirosh I, Bialas AR, Kamitaki N, Martersteck EM, Trombetta JJ, Weitz DA, Sanes JR, Shalek AK, Regev A, McCarroll SA. Highly parallel genome-wide expression profiling of individual cells using nanoliter droplets. Cell. 2015;161:1202-14.

32. Yaari $\mathrm{G}$, Kleinstein SH. Practical guidelines for B-cell receptor repertoire sequencing analysis. Genome Med. 2015;7:121.

33. Lundberg KS, Shoemaker DD, Adams MW, Short JM, Sorge JA, Mathur EJ. High-fidelity amplification using a thermostable DNA polymerase isolated from Pyrococcus furiosus. Gene. 1991;108:1-6.

34. Flaman JM, Frebourg T, Moreau V, Charbonnier F, Martin C, Ishioka C, Friend SH, Iggo R. A rapid PCR fidelity assay. Nucleic Acids Res. 1994;22:3259-60.

35. Zhou Y, Zhang X, Ebright RH. Random mutagenesis of gene-sized DNA molecules by use of PCR with Taq DNA polymerase. Nucleic Acids Res. 1991;19:6052

36. Schirmer M, D'Amore R, ljaz UZ, Hall N, Quince C. Illumina error profiles: resolving fine-scale variation in metagenomic sequencing data. BMC Bioinformatics. 2016:17:125.

37. Andrews KR, Good JM, Miller MR, Luikart G, Hohenlohe PA. Harnessing the power of RADseq for ecological and evolutionary genomics. Nat Rev Genet. 2016;17:81-92.

38. Head SR, Komori HK, LaMere SA, Whisenant T, Van Nieuwerburgh F, Salomon DR, Ordoukhanian P. Library construction for next-generation sequencing: overviews and challenges. Biotechniques. 2014;56:61-4. 66, 68, passim

39. Mathieu-Daudé F, Welsh J, Vogt T, McClelland M. DNA rehybridization during PCR: the 'cot effect' and its consequences. Nucleic Acids Res. 1996; 24:2080-6.

40. Aird D, Ross MG, Chen WS, Danielsson M, Fennell T, Russ C, Jaffe DB, Nusbaum C, Gnirke A. Analyzing and minimizing PCR amplification bias in Illumina sequencing libraries. Genome Biol. 2011;12:R18.

41. Acinas SG, Sarma-Rupavtarm R, Klepac-Ceraj V, Polz MF. PCR-induced sequence artifacts and bias: insights from comparison of two 16S rRNA clone libraries constructed from the same sample. Appl Environ Microbiol. 2005;71:8966-9.

42. Wang GC, Wang Y. Frequency of formation of chimeric molecules as a consequence of PCR coamplification of $16 \mathrm{~S}$ rRNA genes from mixed bacterial genomes. Appl Environ Microbiol. 1997;63:4645-50. 
43. Parekh S, Ziegenhain C, Vieth B, Enard W, Hellmann I. The impact of amplification on differential expression analyses by RNA-seq. Sci Rep. 2016;6:25533.

44. Bansal V. A computational method for estimating the PCR duplication rate in DNA and RNA-seq experiments. BMC Bioinformatics. 2017;18:43.

45. Sayols S, Scherzinger D, Klein H. dupRadar: a Bioconductor package for the assessment of PCR artifacts in RNA-Seq data. BMC Bioinformatics. 2016;17:428.

46. Stegle O, Teichmann SA, Marioni JC. Computational and analytical challenges in single-cell transcriptomics. Nat Rev Genet. 2015;16:133-45.

47. Ziegenhain C, Vieth B, Parekh S, Reinius B, Guillaumet-Adkins A, Smets M, Leonhardt H, Heyn H, Hellmann I, Enard W. Comparative analysis of singlecell RNA sequencing methods. Mol Cell. 2017;65:631-643.e4.

48. Picelli S, Faridani OR, Björklund AK, Winberg G, Sagasser S, Sandberg R. Fulllength RNA-seq from single cells using smart-seq2. Nat Protoc. 2014;9:171-81.

49. Archer N, Walsh MD, Shahrezaei V, Hebenstreit D. Modeling enzyme processivity reveals that RNA-Seq libraries are biased in characteristic and correctable ways. Cell Syst. 2016;3:467-479.e12.

50. Mercer TR, Clark MB, Crawford J, Brunck ME, Gerhardt DJ, Taft RJ, Nielsen LK, Dinger ME, Mattick JS. Targeted sequencing for gene discovery and quantification using RNA CaptureSeq. Nat Protoc. 2014;9:989-1009.

51. Carninci P, Sandelin A, Lenhard B, Katayama S, Shimokawa K, Ponjavic J, Semple CA, Taylor MS, Engström PG, Frith MC, Forrest AR, Alkema WB, Tan SL, Plessy C, Kodzius R, Ravasi T, Kasukawa T, Fukuda S, Kanamori-Katayama M, Kitazume Y, Kawaji H, Kai C, Nakamura M, Konno H, Nakano K, MottaguiTabar S, Arner P, Chesi A, Gustincich S, Persichetti F, Suzuki H, Grimmond SM, Wells CA, Orlando V, Wahlestedt C, Liu ET, Harbers M, Kawai J, Bajic VB, Hume DA, Hayashizaki Y. Genome-wide analysis of mammalian promoter architecture and evolution. Nat Genet. 2006;38:626-35.

52. Park PJ. ChIP-seq: advantages and challenges of a maturing technology. Nat Rev Genet. 2009;10:669-80.

53. Skene PJ, Henikoff S. An efficient targeted nuclease strategy for highresolution mapping of DNA binding sites. eLife. 2017:e21856.

54. Addo-Quaye C, Eshoo TW, Bartel DP, Axtell MJ. Endogenous siRNA and miRNA targets identified by sequencing of the Arabidopsis degradome. Curr Biol. 2008;18:758-62.

55. Morlan JD, Qu K, Sinicropi DV. Selective depletion of rRNA enables whole transcriptome profiling of archival fixed tissue. PLoS One. 2012;7:e42882.

56. Adiconis X, Borges-Rivera D, Satija R, DeLuca DS, Busby MA, Berlin AM, Sivachenko A, Thompson DA, Wysoker A, Fennell T, Gnirke A, Pochet N, Regev A, Levin JZ. Comparative analysis of RNA sequencing methods for degraded or low-input samples. Nat Methods. 2013;10:623-9.

57. Hagberg A, Swart P, Chult DS. Exploring network structure, dynamics, and function using NetworkX, Proceedings of the 7th Python in Science Conference; 2008. p. 11-6.

58. Han BW, Wang W, Zamore PD, Weng Z. piPipes: a set of pipelines for piRNA and transposon analysis via small RNA-seq, RNA-seq, degradomeand CAGE-seq, ChIP-seq and genomic DNA sequencing. Bioinformatics. 2015;31:593-5.

\section{Ready to submit your research? Choose BMC and benefit from:}

- fast, convenient online submission

- thorough peer review by experienced researchers in your field

- rapid publication on acceptance

- support for research data, including large and complex data types

- gold Open Access which fosters wider collaboration and increased citations

- maximum visibility for your research: over $100 \mathrm{M}$ website views per year

At BMC, research is always in progress.

Learn more biomedcentral.com/submissions 